\title{
Shear-induced transitions in a ternary polymeric system
}

\author{
A. V. Zvelindovsky, G. J. A. Sevink, and J. G. E. M. Fraaije \\ Faculty of Mathematics and Natural Sciences, University of Groningen, Nijenborgh 4, 9747 AG Groningen, The Netherlands \\ and Leiden Institute of Chemistry, Leiden University, Einsteinweg 55, 2300 RA Leiden, The Netherlands*
}

(Received 18 November 1999)

\begin{abstract}
The first three-dimensional simulation of shear-induced phase transitions in a polymeric system has been performed. The method is based on dynamic density-functional theory. The pathways between a bicontinuous phase with developing gyroid mesostructure and a lamellar/cylinder phase coexistence are investigated for a mixture of flexible triblock $A B A$ copolymer and solvent under simple steady shear.
\end{abstract}

PACS number(s): 64.70.-p, 61.25.Hq, 61.30.-v, 83.50.Ax

Various self-assembly systems such as lyotropic liquid crystals, surfactants, and block copolymers can form ordered mesophases (lamellar, cylindrical, spherical, etc.). These phases have received much attention because of the fundamental interest in establishing universal laws for selforganization phenomena, and also because of the wide range of applications in materials science [1-6].

An interesting issue in the design of new materials is the modulation of phase behavior by external and internal factors such as flows [7], reactions [8], temperature inhomogeneity [9], confinements, and surfaces [10]. In particular, externally applied shear flows are found to lead to macroscale order in block copolymer systems. Moreover, shear introduces a new kind of phase behavior of block copolymer systems, the so-called orientational phase transitions [11-14].

So far, lamellar, hexagonal cylindrical, and cubic micellar phases of block copolymers under shear have been thoroughly investigated in experiments $[1,7,12,13,15]$. Theoretical understanding is partly reached for the lamellar geometry $[11,16-18]$ and for the hexagonal cylindrical phase $[14,19,20]$. Experimental observation of a sponge phase in low molecular weight surfactants under shear has been carried out recently in [21]. Elongation of mesostructures has been recently found in a sheared isotropic bicontinuous polymer phase [22]. More complex phases such as gyroid and coexistence of phases in shear flow still require experimental examination.

Previously, computer simulations of polymer morphologies under shear have been carried out for lamellar and cylindrical phases in two-dimensional (2D) systems (see references in [1]). Recently, we have reported three-dimensional (3D) density-functional calculations for these two phases $[23,24]$. Here we report on the effect of shear on more complex phases-bicontinuous gyroid and lamellar/cylinder coexistence in a three component copolymer system. We have observed shear-induced transitions between these states. Many shear-induced transitions are found in experiments and theory [1]. The transitions we present here require future experimental and theoretical investigation.

The time evolution of the density field under simple steady shear flow, $v_{x}=\dot{\gamma} y, v_{y}=v_{z}=0$, can be described by a

\footnotetext{
*Leiden University is the present affiliation for all authors since January 2000.
}

time dependent Landau-Ginzburg type equation with a convective term $[4,23,25]$ and a stochastic term $[26,27]$. In general, the velocity field can be found from hydrodynamics. For a system with different viscosities of the components, this leads to an adaption of the linear velocity profile [11]. Such refinement is important for the description of temperature dependency of the orientation of mesostructure lattice in the gradient-vorticity plane at high shear (see discussion in [14]). However, in the weak segregation regime, when there are no steep concentration gradients, the basic features of the process of alignment in flow (regardless of fine details of orientational transitions) can be described accurately by a diffusion-convection equation with an imposed velocity profile $[18,23,24]$.

In contrast to traditional schemes of polymer phase separation dynamics where a Landau Hamiltonian is used with vertex functions calculated following the random phase approximation (see, e.g., [1]), we numerically calculate the "exact" free energy $F$ of a polymer system consisting of Gaussian chains in a mean field environment using path integral formalism $[26,27]$. The benefit of our approach is that it avoids the truncation of the free energy and therefore represents intermediate (metastable) states more accurately.

Some time ago, Matsen and Schick [3] introduced a powerful method for self-consistent-field (SCF) calculations of equilibrium block copolymer morphologies. Our approach uses essentially the same free energy functional and complements as the static SCF calculations by providing a dynamical picture of the system, which is crucial for systems under shear $[4,25,28]$. In our method the calculations are in direct space, without any bias with respect to the morphology formation.

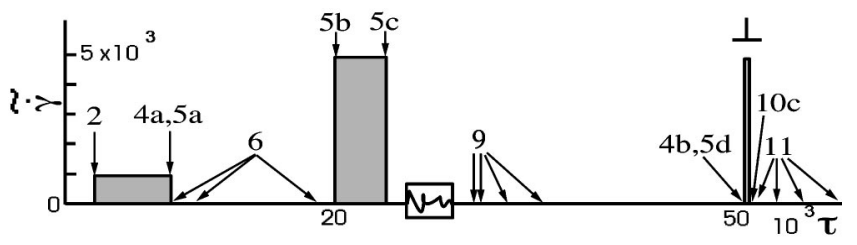

FIG. 1. The sample history. Arrows indicate figures with snapshots. The box with the curve indicates a high noise region and the symbol $\perp$ indicates a change in shear direction. $\tilde{\gamma}=\Delta t \dot{\gamma}$ is the dimensionless shear rate and $\tau$ is dimensionless time with time step $\Delta \tau=\beta^{-1} M h^{-2} \Delta t=0.5$ (see [27]). 


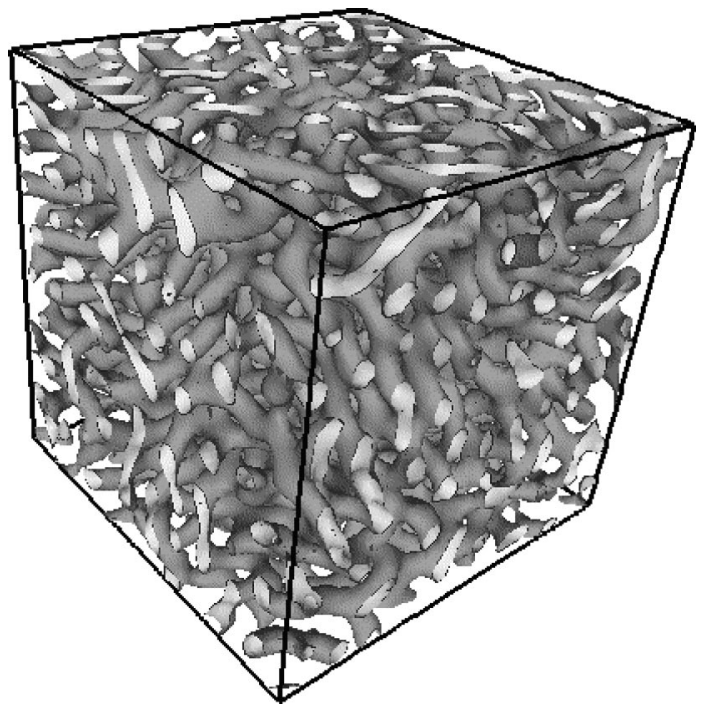

FIG. 2. An isosurface of $P O$ blocks at $\tau=2000$ (no shear).

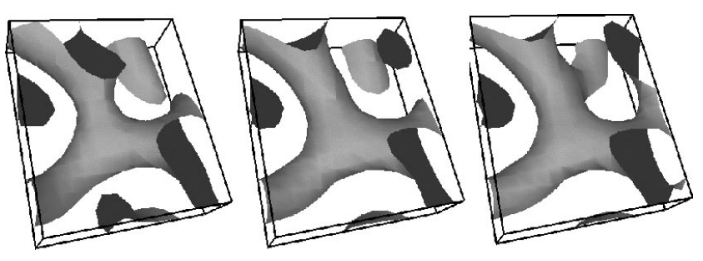

FIG. 3. Evolution of a detail of the bicontinuous morphology in the absence of shear at $\tau=2000,4975,7500$ (from left to right).

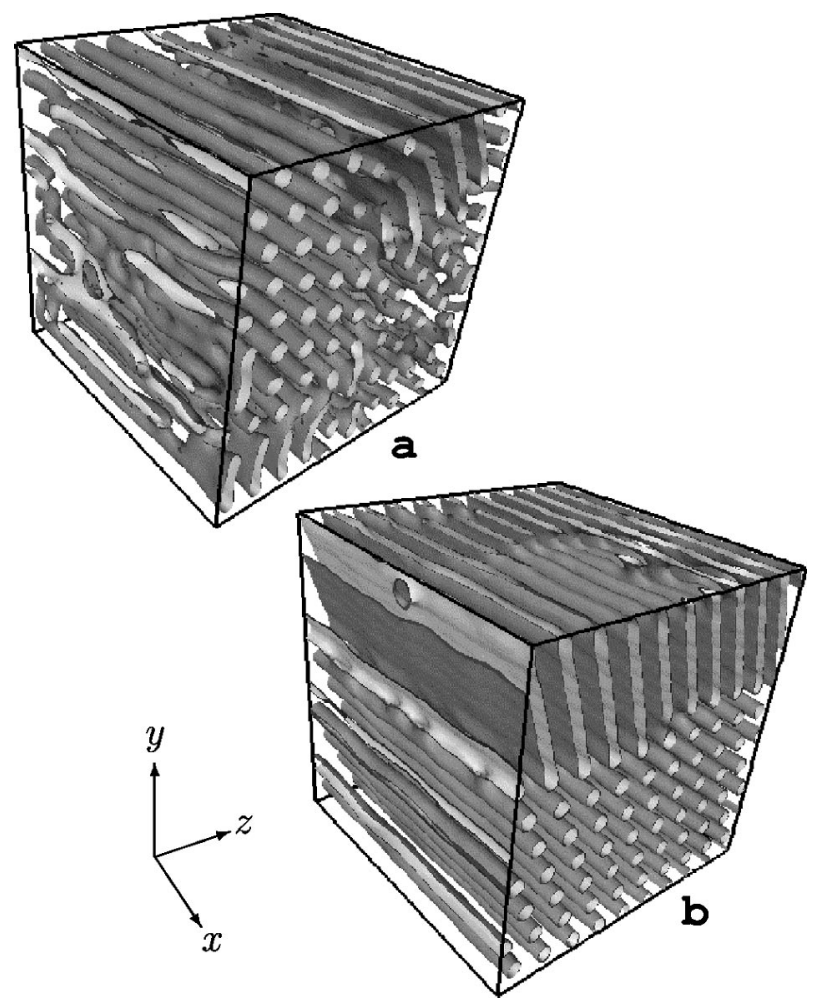

FIG. 4. Morphology of $P O$ blocks at $\tau=7500$ (a) and 50000 (b).

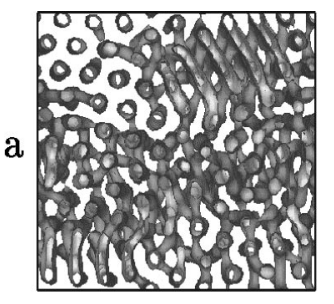

$\mathrm{b}$
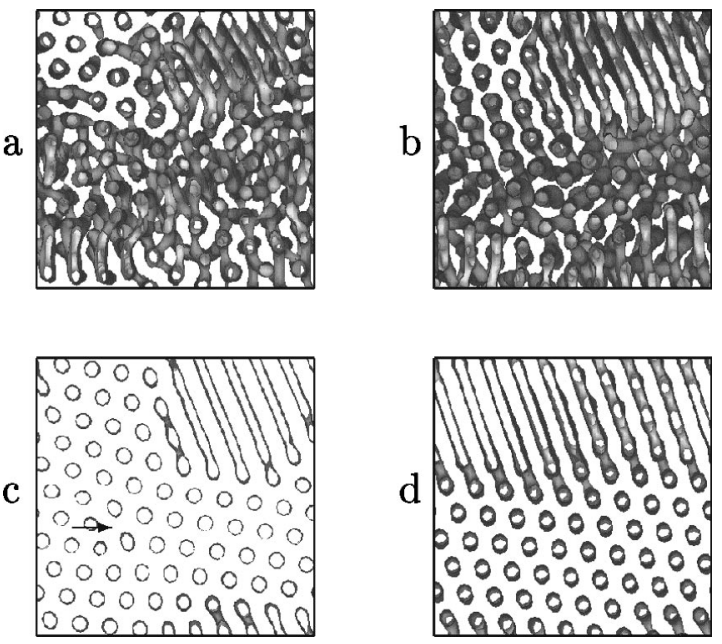

FIG. 5. Orthogonal projection in the $x$ direction of the morphologies at different times: $\tau=7500$ (a), 20000 (b), 23500 (c), 50000 (d). The arrow in (c) denotes a dislocation.
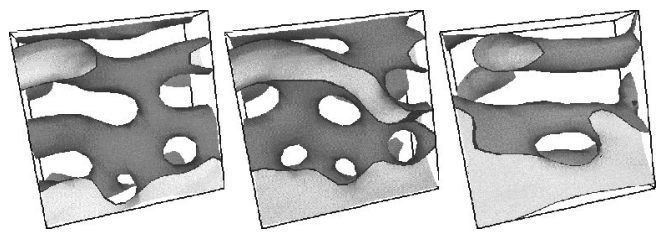

FIG. 6. Detail of a perforated lamellae transformation: $\tau$ $=7500,9500,18500$ (from left to right).
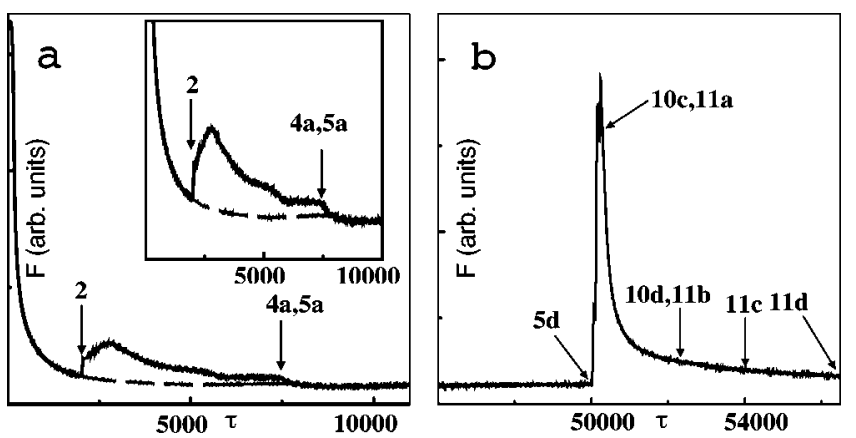

FIG. 7. Free energy as function of the time for the first (a) and the last (b) period of shear. The inset is a magnified representation of the left plot. Arrows indicate figures with snapshots. First and second arrows from the left correspond to starting and stopping times of shearing (cf. Fig. 1). The dashed line on the left graph corresponds to bicontinuous phase evolution in the absence of shear (see Fig. 3).
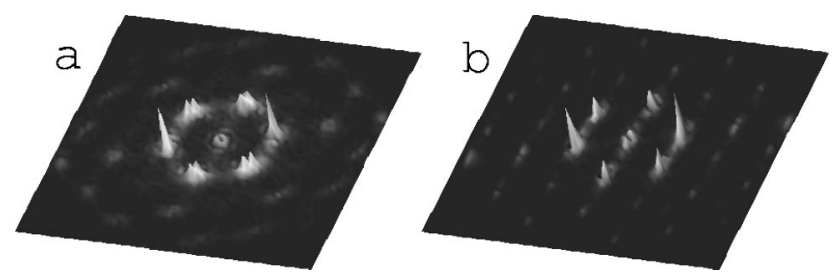

FIG. 8. 3D structure factor summed in the $x$ direction (log scale) for $\tau=23500$ (a) [cf Fig. 5(c)], and $\tau=50000$ (b) [cf. Fig. 5(d)]. 
a

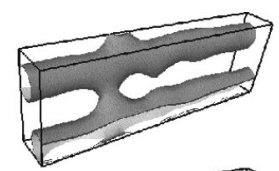

$\mathrm{b}$

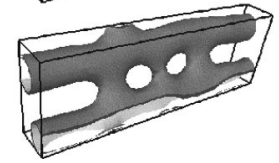

$\mathrm{c}$

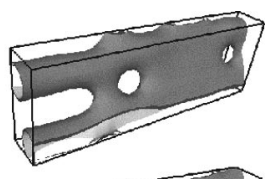

d

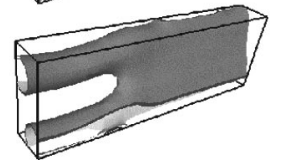

FIG. 9. Detail of neck dynamics: $\tau=30000$ (a), 30500 (b), 32500 (c), 35000 (d).

The system presented here is a mixture of Gaussian chains $E_{3} P_{9} E_{3}$ (bead names are arbitrary) and solvent that is parameterized to model $60 \%$ aqueous solution of a triblock copolymer Pluronic surfactant PL64, consisting of ethylene oxide and propylene oxide blocks $(E O)_{13}(P O)_{30}(E O)_{13}$ with the hydrophobic block in the middle [27]. The choice is justified by a huge variety of available experimental data. In an experimental phase diagram, $60 \%$ polymer concentration corresponds to a very complex phase coexistence region [29].

Figure 1 gives a schematic overview of the simulation of the system in a 3D box $(64 \times 64 \times 64)$. The starting configuration is a homogeneous distribution of the components. The system (Fig. 2) demonstrates the development of a bicontinuous morphology with clear gyroid-type connectivity (Fig. 3); however, it is still without global symmetry throughout the sample. The system can remain in this phase a very long time, slowly rearranging the structure and keeping gyroidtype connectivity [see also Fig. 7(a)].

After applying shear $\left(\tilde{\gamma}=10^{-3}\right)$ to the morphology shown in Fig. 2, the system slowly deforms. However, the connectivity hardly changes: the system remains bicontinuous and similar to the morphology shown in Fig. 2 even at large shear strains $\gamma>1(100 \%)$. While shearing continues, connections in the system start to break, and separate pieces of the structure reconnect to form a pattern aligned in the flow
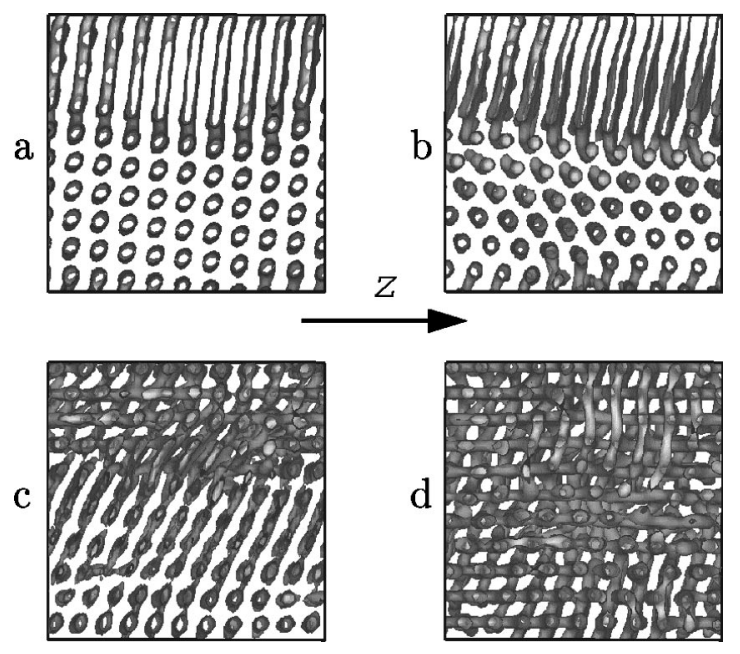

FIG. 10. Results of shear in the $z$ direction, taking the morphology as shown in Fig. 4(b) $(\tau=50000)$, as starting structure. Orthogonal projections of the morphologies at $\tau=50050$ (a), the end of shear, and $\tau=54050$ (b). A longer period of shear from the same starting structure: $\tau=50300$ (c), the end of shear, and at $\tau$ $=52300(\mathrm{~d})$.
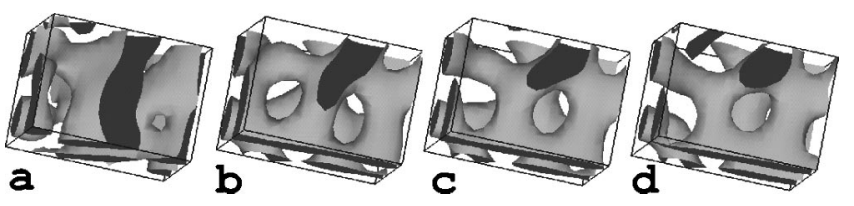

FIG. 11. Detail of structure relaxation after shearing in the $z$ direction at $\tau=50300$ (a) [cf. Fig. 10(c)], 52300 (b) [cf Fig. 10(c)], 54000 (c), 56500 (d).

direction. New structure consists of coexisting lamellae and hexagonally packed cylindrical clusters, as shown in Figs. 4(a) and 5(a).

Stopping the shear at some point $[\tau=7500(\gamma=11)$, Fig. 1] leads to the reorganization of the structure via migration of defects (holes in lamellae, necks between cylinders); see Fig. 6. The time evolution of deformed and broken bicontinuous structure involves a lot of perforated lamellar clusters. Migration of defects in these clusters sometimes leads to intermediate structures with a locally hexagonal-like arrangement of holes (Fig. 6, middle picture). After long relaxation without shear, the structure remains a coexistence of defected cylinders and lamellae [Fig. 5(b)] but with a better ordering compared to the moment of stopping shear [Fig. 5(a)]. The free energy plot (Fig. 7) provides information about which state is metastable. Bicontinuous and coexistence states are very close in free energy value in the plateau region [Fig. 7(a)], but the latter is slightly lower.

A second period of stronger shear $\left(\tilde{\gamma}=5 \times 10^{-3}\right)$ very quickly breaks up the remaining connections, and the system flows as a lamellar/cylinder coexistence without changing lamellar/cylinder volume ratio while shear continues; $\tau$ $=23500(\gamma=35)$, Fig. 5(c). The lamellae have only a few holes. The cylinder region no longer has "neck" defects and the only remaining defect is a long living dislocation that remains stable during the whole period of shearing. As a result, the hexagonally packed cylinders consist of two clusters with different internal hexagonal orientation [as can also be seen from the double peaks in the structure factor, Fig. 8(a)]. After stopping shear at $\gamma=35$ [Fig. 5(c)] the system relaxes to a lamellar/cylinder coexistence in which the hexagonal lattice has only one orientation [Figs. 4(b), 5(d), and 8(b)]. Undulated cylinders partly transform into lamellae via formation of subsequent necks; see Fig. 9. The lamellar phase still consists of perforated lamellae, with a low fraction of holes. This coexistence of phases seems to be stable and even the application of a much higher noise $(\Omega=10$ [27]; the region is schematically shown in Fig. 1) does not change the picture considerably.

Both periods of shear demonstrate that the lamellar cluster becomes larger after shear is released [Figs. 5(a)-5(d)]. This is consistent with the fact that switching on the shear again squeezes the size of the lamellar region [Figs. 5(b) and 5(c)].

The stability of the phase coexistence morphology, Figs. 4(b), and $5(\mathrm{~d})$, is challenged by applying shear $(\tilde{\gamma}=5$ $\times 10^{-3}$ ) in the $z$ direction (perpendicular to lamellae/ cylinders); see Fig. 1. In Figs. 10(a) and 10(c) we see the result of shearing for a period of $\tau=50(\gamma=0.5)$ and $\tau$ $=300(\gamma=3)$, respectively. While shearing, the lamellae start to tilt and thin. The cylinders start to roll over each other and deform to a slightly prolate shape in cross section. 
This leads to an energetically unfavorable cubic cylindrical lattice, Fig. 10(a), or to a morphology with very oblong cylinders in cross section and partly broken lamellae, Figs. 10(c) and 11(a). Releasing shear at these different stages leads to different phenomena. After a relatively small distortion of the system $[\gamma=0.5$; Fig. $10(a)]$ in short time the system relaxes back from cubic to hexagonal packed cylinders, keeping the lamellar cluster intact; see Fig. 10(b). However, if shear is stopped after a larger distortion $[\gamma=3$; Fig. 10(c)], then very quickly, necks form throughout the sample; see Fig. 10(d). The structure becomes bicontinuous with sometimes obviously gyroidlike connectivity; see Fig. 11(c). However, the global structure is different from the initial morphology in Fig. 2. The process of relaxation after stopping shear goes via the following stages: frustrated lamellae/ cylinders $\rightarrow$ bicontinuous phase $\rightarrow$ lamellae/cylinders. The first stage is relatively fast whereas the last is slow, which can be viewed in hardly noticeable changes in structure in Figs. 11(c) and 11(d). The free energy plot [Fig. 7(b)] also demonstrates that the system tends to relax back to a coexistence state. Thus in this case the bicontinuous phase is a long living intermediate stage with the free energy very close to the lamellae/cylinders coexistence state.

In summary, we have performed the first 3D shear simulation of a bicontinuous $A B A$ copolymer/solvent system. The shear-induced phase transitions from the bicontinuous phase to lamellae/cylinder coexistence and back have been detected. These two states have very close free energy values and can be separated by a barrier in the free energy landscape. As a result, the polymeric system can be trapped in either of them.

A. V. Z. and G. J. A. S. acknowledge support of the MesoDyn project ESPRIT No. EP22685 of the European Community. We acknowledge support of NCF (Stichting Nationale Computer Faciliteiten).
[1] I. W. Hamley, The Physics of Block Copolymers (Oxford University Press, Oxford, 1998).

[2] F. S. Bates and G. H. Fredrickson, Phys. Today 52 (2), 32 (1999)

[3] M. W. Matsen and M. Schick, Phys. Rev. Lett. 72, 2660 (1994)

[4] H. Kodama and M. Doi, Macromolecules 29, 2652 (1996).

[5] S. T. Milner and P. D. Olmsted, J. Phys. II 7, 249 (1997).

[6] S. Qi and Z.-G. Wang, Phys. Rev. Lett. 76, 1679 (1996); Phys. Rev. E 55, 1682 (1997).

[7] Z.-R. Chen, A. M. Issaian, J. A. Kornfield, S. D. Smith, J. T. Grothaus, and M. M. Satkowski, Macromolecules 30, 7096 (1997).

[8] N. M. Maurits, G. J. A. Sevink, A. V. Zvelindovsky, and J. G. E. M. Fraaije, Macromolecules 32, 70674 (1999).

[9] J. Bodycomb, Y. Funaki, K. Kimishima, and T. Hashimoto, Macromolecules 32, 2075 (1999).

[10] G. J. A. Sevink, A. V. Zvelindovsky, B. A. C. van Vlimmeren, N. M. Maurits, and J. G. E. M. Fraaije, J. Chem. Phys. 110, 2250 (1999).

[11] G. H. Fredrickson, J. Rheol. 38, 1045 (1994).

[12] U. Wiesner, Macromol. Chem. Phys. 198, 3319 (1997).

[13] T. Tepe, M. F. Schulz, J. Zhao, M. Tirrell, and F. S. Bates, Macromolecules 28, 3008 (1995).

[14] A. N. Morozov, A. V. Zvelindovsky, and J. G. E. M. Fraaije, Phys. Rev. E 61, 4125 (2000).

[15] G. Schmidt, W. Richtering, P. Lindner, and P. Alexandridis, Macromolecules 31, 2293 (1998).

[16] M. E. Cates and S. T. Milner, Phys. Rev. Lett. 62, 1856 (1989).

[17] M. Goulian and S. T. Milner, Phys. Rev. Lett. 74, 1775 (1995).
[18] F. Drolet, P. Chen, and J. Viñals, Macromolecules 32, 8603 (1999).

[19] C. M. Marques and M. E. Cates, J. Phys. (France) 51, 1733 (1990).

[20] C.-Y. Huang and M. Muthukumar, J. Chem. Phys. 107, 5561 (1997).

[21] H. F. Mahjoub, C. Bourgaux, P. Sergot, and M. Kleman, Phys. Rev. Lett. 81, 2076 (1998).

[22] F. Qiu, J. Ding, and Y. Yang, Phys. Rev. E 58, R1230 (1998).

[23] A. V. Zvelindovsky, G. J. A. Sevink, B. A. C. van Vlimmeren, N. M. Maurits, and J. G. E. M. Fraaije, Phys. Rev. E 57, R4879 (1998); Prog. Colloid Polym. Sci. 110, 251 (1998).

[24] A. V. M. Zvelindovsky, B. A. C. van Vlimmeren, G. J. A. Sevink, N. M. Maurits, and J. G. E. M. Fraaije, J. Chem. Phys. 109, 8751 (1998).

[25] A. Onuki, J. Phys.: Condens. Matter 9, 6119 (1997).

[26] J. G. E. M. Fraaije, J. Chem. Phys. 99, 9202 (1993); 100, 6984 (1994); J. G. E. M. Fraaije, B. A. C. van Vlimmeren, N. M. Maurits, M. Postma, O. A. Evers, C. Hoffmann, P. Altevogt, and G. Goldbeck-Wood, ibid. 106, 4260 (1997).

[27] B. A. C. van Vlimmeren, N. M. Maurits, A. V. Zvelindovsky, G. J. A. Sevink, and J. G. E. M. Fraaije, Macromolecules 32, 646 (1999). The used parameters are $\chi_{E O, \omega}=1.4, \chi_{P O, \omega}$ $=1.7, \chi_{E O, P O}=3, \Omega=100, d=1.1543, \kappa^{\prime}=10$. The noise scaling parameter is $\Omega=\nu^{-1} h^{3}$, where $\nu$ is a bead volume parameter and $h$ is a mesh size of a cubic grid. For the described system, $\Omega$ lies in the region $10-100$. This parameter is related to a number of "degrees of freedom" in a coarsegrained cell (see the above article for more extensive discussion).

[28] T. Kawakatsu, Phys. Rev. E 56, 3240 (1997); 57, 6214 (1998).

[29] P. Alexandridis, U. Olsson, and B. Lindman, Macromolecules 28, 7700 (1995). 